\title{
Design and in vitro characterization of a topical nanoemulsion- enriched hydrogel of econazole nitrate
}

\author{
Mowafaq M. Ghareeb \\ Department of Pharmaceutics, College of Pharmacy, University of Baghdad, Baghdad, Iraq.
}

\begin{tabular}{l}
\hline ARTICLE INFO \\
\hline Received on: $21 / 08 / 2018$ \\
Accepted on: $06 / 12 / 2018$ \\
Available online: $31 / 01 / 2019$
\end{tabular}

Key words:

Econazole nitrate,

Nanoemulsion-enriched

hydrogel.

\begin{abstract}
Background/Objective: The aim of the study was to prepare a nanoemulsion (NE)-enriched hydrogel of econazole nitrate (EC) for a topical broad-spectrum antimycotic application. EC is classified as a Biopharmaceutical Classification System Class IV (low permeability, low solubility) drug. Nanotechnology is one of the most important approaches to improve drugs solubility and permeability.

Methods: Depending on solubility study of EC in NE components which include oils (peppermint oil, eucalyptus oil, and olive oil), surfactants (Tween 40, Brij 35, and Ceto stearyl alcohol), and co-surfactants (Propylene glycol, PEG 400, and Glycerol); peppermint oil was selected as the oil phase. Tween 40 and propylene glycol were selected as the surfactant and co-surfactant, respectively. Deionized water was used as an aqueous phase. A phase diagram was prepared with a 5:1 weight ratio of tween 40 to propylene glycol. One percent of EC was loaded into the selected NE system and combined with hydrogel consisting of carbopol 934 or HPMC as gelling polymer at three different percentages. Evaluation and in vitro release of the product were done.

Results: NE Formula (F10) which is composed of $10 \% \mathrm{w} / \mathrm{w}$ of each the oil and surfactant mixture was selected as optimum NE which shows a particle size of $80.57 \mathrm{~nm}$ and acceptable viscosity and $\mathrm{pH}$ (5.65). Formula (G6) which is composed of NE (F10) combined with hydrogel 3.5\% HPMC shows satisfactory physical properties with the complete and prolonged release over 8 hours.

Conclusion: The results suggest that the prepared NE-enriched hydrogel can be considered as a promising delivery system for class IV antifungal drug.
\end{abstract}

\section{INTRODUCTION}

Econazole nitrate (EC) is a broad-spectrum antimycotic imidazole derivative agent with fungistatic action and is widely used against Candida albicans (Ogata et al., 1983). According to the Biopharmaceutical Classification System, econazole nitrate is classified as a Class IV (low permeability, low solubility) drug (Amidon et al., 1995). It is very slightly soluble in water $(1.48 \mu \mathrm{g} / \mathrm{ml})$ but soluble in methanol (Suñer-Carbó et al., 2017). It has a partition coefficient $(\log P)$ of about 5.5 and a pKa of 6.6 (Remington, 2006; Stahl and Wermuth, 2002). One of the commercial topical dosage

"Corresponding Author

Mowafaq M. Ghareeb, Department of Pharmaceutics, College of

Pharmacy, University of Baghdad, Baghdad, Iraq.

E-mail:mopharmacy@yahoo.com; mopharmacy@gmail.com forms of EC is a cream containing $1 \%$ of EC. This cream provides neither a complete penetration of EC nor a long residence time of the formulation on the infected area (Ge et al., 2014). Most of the topical dermatological formulations such as creams and ointments have the disadvantages of a less spreading coefficient, sticky nature, and the need for rubbing during application. These limitations can be eliminated through a hydrogel formulation. Despite various advantages of hydrogels, they have a major limitation in the delivery of hydrophobic drugs. Thus, nanoemulsion (NE) combined with hydrogel has proved a boon in the delivery of hydrophobic drugs topically and provides the advantages of hydrogel formulation (Hoare and Kohane, 2008).

$\mathrm{NE}$ is a transparent isotropic, heterogeneous system of two immiscible liquids consisting of a fine dispersion of drugs in nanodroplets. It is stabilized by an interfacial layer of surfactant and co-surfactant (Sood et al., 2014). NEs are kinetically and 
thermodynamically stable systems lacking any coalescence or apparent flocculation upon storage and use within the period of the expiration date. NEs with nanodroplet size in the range of 20-400 $\mathrm{nm}$, uniform droplet size distribution commonly have different biopharmaceutical properties comparing to the traditional emulsions type which have particle size higher than $500 \mathrm{~nm}$ (Ahmed et al., 2012; Shakeel et al., 2012; Sugumar et al., 2014). NEs show many advantages comparing to conventional emulsions, such as more stability, higher interfacial area, fast absorption by internalization into the enterocyte, enhancement of drug aqueous solubility, and consequently, expected improvement in bioavailability (Gorain et al., 2014). US Food and Drug Administration has approved a number of NEs in the past few years such as $\mathrm{Norvir}^{\circledR}$, Neoral ${ }^{\circledR}$, and Fortovase ${ }^{\circledR}$ (Zhao et al., 2013). The aim of this study was to control the delivery of econazole nitrate topically with fast and easy permeation through skin epidermis by incorporating the drug in an NE combined with hydrogel to provide more residence time of drug at the infected site with optimum skin penetration.

\section{MATERIALS AND METHODS}

\section{Materials}

Econazole nitrate was generously supplied by Furat pharmaceutical industries, Iraq. Peppermint oil was purchased from Bar-sur-Loup Grasse (a.m) France, eucalyptus oil and olive oil were purchased from Ati lab., Netherland. Tween 40 was purchased from Grin land chemical comp., UK. Brij 35 was purchased from Himedia Lab. Pvt Ltd, India. Cetostearyl alcohol was purchased from Fluca Chemie AG, Switzerland. All other chemicals were of analytical grade.

\section{Methods}

\section{Differential scanning calorimetry}

The sample of the drug was analyzed by differential scanning calorimetry (DSC) using a Mettler Toledo SR system. Three mg of each sample was placed into a pierced aluminum container. The study was performed under the static air atmosphere in the temperature range of $20^{\circ} \mathrm{C}-320^{\circ} \mathrm{C}$ at a heating rate of $10^{\circ} \mathrm{C} /$ minute. The peak temperature was determined.

\section{HPLC analysis of econazole nitrate}

To determine the drug content, the Reverse phase (RP)-high-pressure liquid chromatographic (HPLC) separation was performed on a Shimadzu liquid chromatographic system equipped with an LC pump, DGU-20A5 UV detector, and injector system fitted with $100 \mu 1$ loop volume. The HPLC analysis was carried out at a flow rate of $2.0 \mathrm{ml} /$ minute using a mobile phase composed of methanol-aqueous $0.05 \mathrm{M}$ ammonium dihydrogen phosphate $(85: 15, \mathrm{v} / \mathrm{v})$, and the UV-detector was set at $230 \mathrm{~nm}$. The mobile phase was prepared daily, filtered through a 0.45 $\mu \mathrm{m}$ membrane filter (Millipore), and sonicated before use. A Thermo C18 column $(100 \mathrm{~mm} \times 4.6 \mathrm{~mm}$ id, $3.5 \mu)$ was used for the separation.

The retention time of drug is 4.78 minutes, as shown in the chromatogram in Figure 1 and drug content was calculated from the calibration curve $(y=0.7354 x+0.00865)$ (Cavrini et al., 1982).

\section{Determination of econazole nitrate solubility in NE components}

Powder of EC was added in excess to different NE components which include oils (peppermint oil, eucalyptus oil, and olive oil), surfactants (Tween 40, Brij 35, and cetostearyl alcohol), and co-surfactants (propylene glycol, PEG 400, and glycerol). After shaking at $25^{\circ} \mathrm{C}$ for 24 hours, samples were centrifuged, and then the supernatant was separated and subjected to centrifugation at 3,000 rpm for 5 minutes to remove the undissolved drug. Samples of these solutions were then collected, and the drug concentration was determined using the HPLC method described here.

\section{Construction of pseudo-ternary phase diagram}

To find out the concentration of components that produce NEs, pseudo-ternary phase diagrams were constructed using water titration method at an ambient temperature $\left(25^{\circ} \mathrm{C}\right)$. Peppermint oil was selected as the oil phase. Tween 40 and propylene glycol were selected as surfactant and co-surfactant, respectively. Deionized water was used as an aqueous phase. A phase diagram was prepared with a 5:1 weight ratio of tween 40 to propylene glycol.

Fourteen formulas of NE were prepared at different weight ratios, as shown in Table 1. The mixtures of oil, surfactant, and co-surfactant at certain weight ratios were diluted with

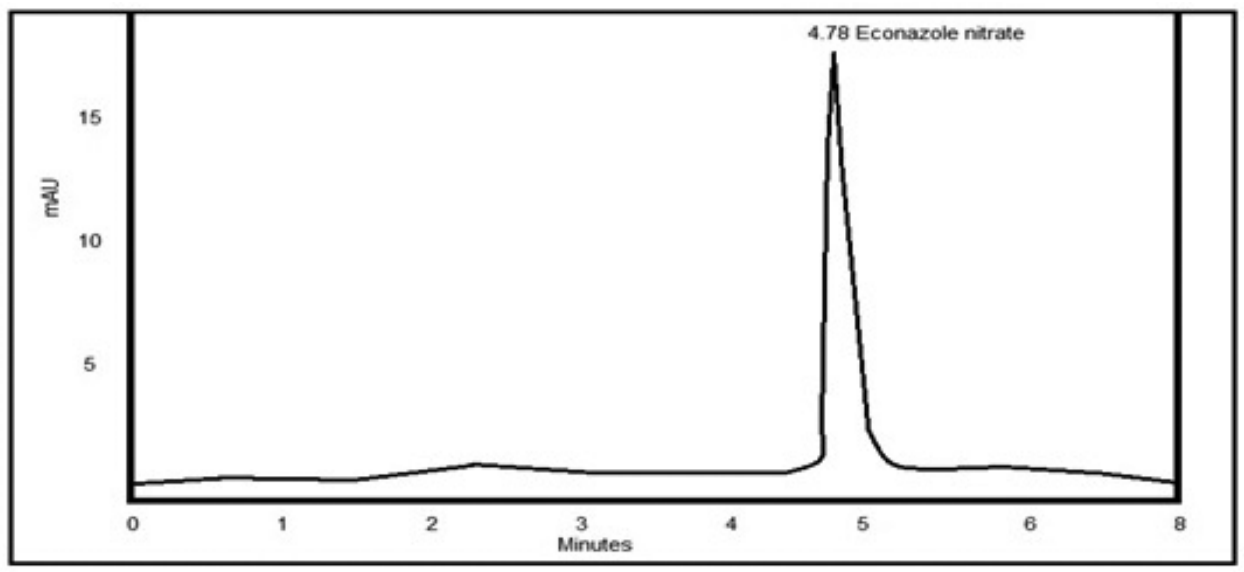

Figure 1. HPLC chromatogram of econazole nitrate. 
water dropwise under moderate magnetic stirring. After being equilibrated, the mixtures were assessed visually to determine it is one phase NEs (Jadhav et al., 2015).

\section{Drug loading in the NE system}

The loading procedure of $1 \% \mathrm{w} / \mathrm{w}$ EC into the selected NE system includes heating of all the system components, except water up to $40^{\circ} \mathrm{C} \pm 5^{\circ} \mathrm{C}$ using a water bath. Then, the drug is added and the system is subjected to sonication for about 30 minutes at $40^{\circ} \mathrm{C} \pm 5^{\circ} \mathrm{C}$ using a Copley Scientific sonicator $2200 \mathrm{E}$, UK to ensure complete dissolving of the powder.

After ensuring a complete dissolving of all the molecules of the drug visually, the NE system is cooled down to about $30^{\circ} \mathrm{C}$ $\pm 5^{\circ} \mathrm{C}$ and then completed by adding the required weight of water up to $100 \mathrm{~g}$.

\section{Preparation of nanoemulsion-enriched hydrogel of Econazole nitrate}

After loading of drug into the best-chosen nanoemulsion (EC NE), the hydrogel part preparation involves either Carbopol 934 or HPMC as a gelling agent in addition to methyl and propylparaben as a preservative, while propylene glycol as a cosolvent, as shown in Table 2.

The Carbopol 934 gel bases were prepared by dispersing Carbopol 934 in deionized water with a continuous stirring at a moderate speed using a mechanical shaker (Formulations G1, G2, and G3). The $\mathrm{pH}$ of all the formulations was adjusted to 6-6.5 using tri-ethanolamine (Ranga et al., 2012).

In formulations $\mathrm{G} 4, \mathrm{G} 5$, and $\mathrm{G} 6$, the gel was prepared by dispersing HPMC in hot distilled water $\left(80^{\circ} \mathrm{C}\right)$ and the dispersion was cooled and left overnight.

Methyl and propylparaben were dissolved in propylene glycol and mixed with the aqueous phase. Both the $\mathrm{NE}$ and aqueous phases were heated to $70^{\circ} \mathrm{C}-80^{\circ} \mathrm{C}$ separately; then the NE was added to the aqueous phase with continuous stirring until the system temperature drops down to room temperature. The obtained NE was mixed with the hydrogel in 1:1 ratio with gentle stirring to obtain the NE-enriched hydrogel.

\section{Determination of particle size of nanoemulsions}

The particle size and polydispersity were determined for the prepared NE three times by the use of Angstrom nano laser particle size analyzer (Guttoff et al., 2015).

\section{Determination of viscosity of nanoemulsions}

The viscosities of the prepared NEs were determined using ProRheo Viscometer ProRheo, Bell technology Ltd, New Zealand spindle 01 at $50 \mathrm{rpm}$ and room temperature $\left(25^{\circ} \mathrm{C}\right)$ (Guttoff et al., 2015).

Table 1. Composition of econazole nitrate nanoemulsion.

\begin{tabular}{|c|c|c|c|c|}
\hline \multirow{3}{*}{ Formula code } & \multicolumn{3}{|c|}{ The ratio of surfactant to co-surfactant $=5: 1$} & \multirow{3}{*}{$\begin{array}{c}\text { Total nanoemulsion weight } \\
\text { (g) }\end{array}$} \\
\hline & \multicolumn{3}{|c|}{ Nanoemulsion composition as weight ratio } & \\
\hline & surfactant/co-surfactant mixture & oil & water & \\
\hline F1 & 1 & 10 & 89 & 100 \\
\hline $\mathrm{F} 2$ & 2 & 10 & 88 & 100 \\
\hline F3 & 3 & 10 & 87 & 100 \\
\hline F4 & 4 & 10 & 86 & 100 \\
\hline F5 & 5 & 10 & 85 & 100 \\
\hline F6 & 6 & 10 & 84 & 100 \\
\hline F7 & 7 & 10 & 83 & 100 \\
\hline F8 & 8 & 10 & 82 & 100 \\
\hline F9 & 9 & 10 & 81 & 100 \\
\hline F10 & 10 & 10 & 80 & 100 \\
\hline F11 & 20 & 10 & 70 & 100 \\
\hline $\mathrm{F} 12$ & 30 & 10 & 60 & 100 \\
\hline F13 & 40 & 10 & 50 & 100 \\
\hline F14 & 50 & 10 & 40 & 100 \\
\hline
\end{tabular}

Table 2. Formulas of econazole nitrate nanoemulsion-enriched hydrogel.

\begin{tabular}{lcccccc}
\hline Material(g) & G1 & G2 & G3 & G4 & G5 & G6 \\
\hline EC NE & 50 & 50 & 50 & 50 & 50 & 50 \\
Carbopol 934 & 0.5 & 1 & 1.5 & & & \\
HPMC & & & & 1.5 & 2.5 & 3.5 \\
Propylene glycol & 5 & 5 & 5 & 5 & 5 & 5 \\
Methylparaben & 0.03 & 0.03 & 0.03 & 0.03 & 0.03 & 0.03 \\
Propylparaben & 0.01 & 0.01 & 0.01 & 0.01 & 0.01 & 0.01 \\
Water up to & 100 & 100 & 100 & 100 & 100 & 100 \\
\hline
\end{tabular}




\section{Determination of $\mathrm{pH}$ of nanoemulsions}

The $\mathrm{pH}$ values of the prepared NE samples were measured by a $\mathrm{pH}$ meter, Italy at room temperature, $\mathrm{pH}$ buffer solutions $(4.01 \pm 0.02$ and $9.21 \pm 0.02)$ were used to calibrate the instrument, and the measurements were carried out in triplicates (Guttoff et al., 2015).

\section{Physical appearance of nanoemulsion-enriched hydrogel}

The prepared hydrogel formulations were inspected visually for their color, homogeneity, consistency, grittiness, and phase separation.

\section{pH of nanoemulsion-enriched hydrogel}

One gram of the hydrogel formulation was dispersed in $10 \mathrm{ml}$ of distilled water, and a digital pen $\mathrm{pH}$ meter was used to determine the $\mathrm{pH}$ value.

\section{Viscosity of nanoemulsion-enriched hydrogel}

The viscosities of all the prepared hydrogel formulas were measured using ProRheo Viscometer ProRheo, Bell technology Ltd, New Zealand spindle 02 and speed $50 \mathrm{rpm}$ at $25^{\circ} \mathrm{C}$, measurements were carried out in triplicate, and the average value was calculated (Baibhav et al., 2012).

\section{Spreadability of nanoemulsion-enriched hydrogel}

The apparatus suggested by Kumar and Verma (2010) was used to determine the spreadability. It consists of a wooden block, which is attached to a pulley at one end. Spreadability was measured on the basis of "Slip" and "Drag" characteristics of the hydrogel. A ground glass slide was fixed on the wooden block. An excess of hydrogel (about $1 \mathrm{~g}$ ) under study was placed on this ground slide.

The hydrogel preparation was then sandwiched between this slide and second glass slide having the same dimension as that of the fixed ground slide. The second glass slide is provided with the hook.

A weight of $500 \mathrm{mg}$ was placed on the top of the two slides for 5 minutes. The diameter of the produced circle was measured and used as comparative values for spreadability. It is calculated by using the following formula.

$$
\text { Spreadability }=W \cdot D / T
$$

Where, $W=$ wt. on the upper slide; $D=$ diameter of spreading gel; and $T=$ time until spreading stopped (Kumar and Verma, 2010).

\section{Extrudability of nanoemulsion-enriched hydrogel}

It is a common test to measure the force required to extrude the material from the tube. The extrudability test was carried out using $30 \mathrm{~g}$ of hydrogel filled in an aluminum tube. The plunger was adjusted to hold the tube properly. The force of $1 \mathrm{~kg}$ / $\mathrm{cm}^{2}$ was applied for 30 seconds. The quantity of gel extruded was weighed. The procedure was repeated at three equidistance places of the tube (Vikrant and Sonali, 2013).

\section{Determination of drug content of nanoemulsion-enriched hydrogel}

An accurately weighted portion from prepared NEenriched hydrogel of econazole nitrate was dissolved in methanol, and this solution was filtered and the drug content was determined by RP-HPLC method.

\section{In vitro drug release from nanoemulsion-enriched hydrogel}

A sample of $1 \mathrm{~g}$ of the preparation was spread on a filter paper. The hydrogel-loaded membrane was firmly stretched over the edge of a glass tube of $2 \mathrm{~cm}$ diameter (opened at both ends). The membrane was tied up with a rubber. The tube was then immersed in the dissolution vessel, which contained $500 \mathrm{ml}$ of the release medium, $\mathrm{PBS} \mathrm{pH} \mathrm{7.4,} \mathrm{and} \mathrm{maintained} \mathrm{at} 32^{\circ} \mathrm{C} \pm 0.5^{\circ} \mathrm{C}$. The medium was stirred at $50 \mathrm{rpm}$ and 5-ml aliquots were withdrawn from the solution at specified time intervals. Equal volumes of fresh release medium were used to replace the withdrawn samples. The samples were assayed using the HPLC method mentioned previously. Each data point represents the average of three determinations. In vitro release studies were recorded for a period of 8 hours (Abdul Rasool et al., 2011).

\section{RESULTS AND DISCUSSION}

\section{Differential scanning calorimetry}

The results in Figure 2 obtained from DSC analysis showed an endothermic peak of $\mathrm{EN}$ at $165.32^{\circ} \mathrm{C}$ and exothermic peak at $199.32^{\circ} \mathrm{C}$ and these results similar to the published papers which indicate the purity of raw material (El et al., 2017).

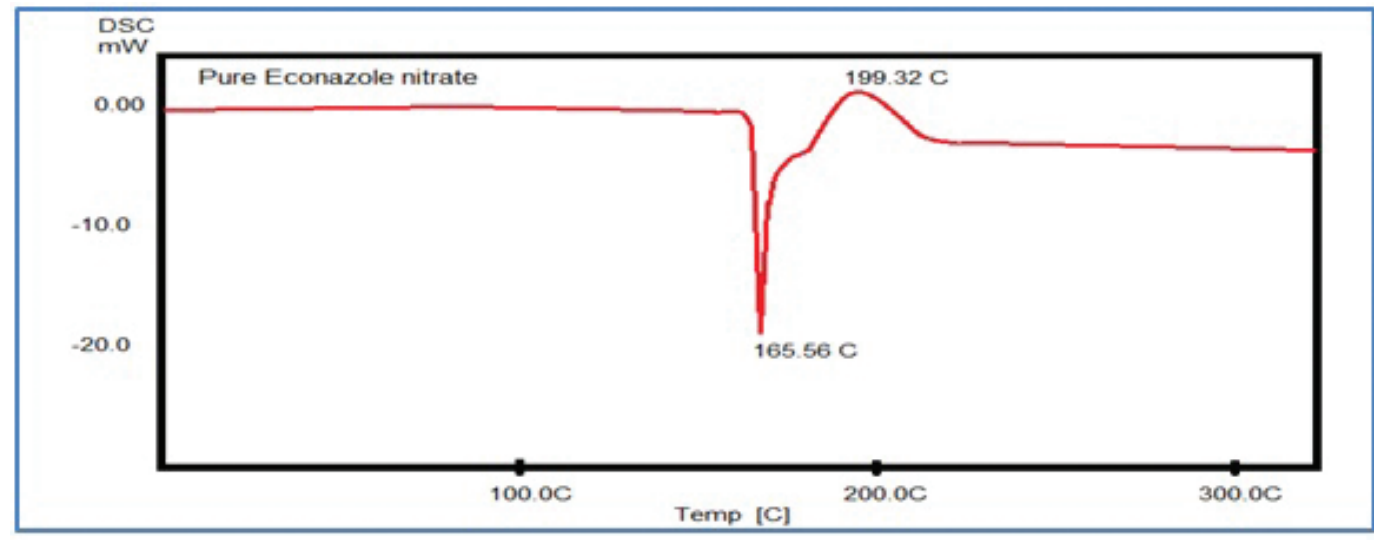

Figure 2. DSC thermogram of econazole nitrate. 


\section{Econazole nitrate solubility in $\mathrm{NE}$ components}

The solubility EC in oil listed in Table 3 was in the following order; Peppermint oil $>$ Eucalyptus oil $>$ Olive oil, while the solubility in surfactant was in the following descending order; Tween $40>$ Brij $35>$ Cetostearyl alcohol. On the other hand, the solubility of EC in co-surfactant was in the following descending order; Propylene glycol > PEG $400>$ Ethanol. So, suggested a system of NE is peppermint oil: Tween 40/propylene glycol: water with the $5: 1$ weight ratio of tween 40 to propylene glycol.

\section{Pseudo-ternary phase diagram}

The constructed pseudo-ternary phase diagram (Fig. 3) shows that formulas F10-F14 produce one phase NE and physical characteristics listed in Table 4. The droplet sizes for the formulations are represented in Table 4. The result shows that the droplet diameter and polydispersity index decrease with increasing surfactant mixture ratio in the system. These

Table 3. Solubility of econazole nitrate in nanoemulsion components.

\begin{tabular}{lc}
\hline Nano-emulsion components & EC solubility $\mathbf{~ m g} \mathbf{m l}$ \\
\hline Oils & 82 \\
Peppermint oil & 57 \\
Eucalyptus oil & 6.85 \\
Olive oil & \\
Surfactant & 3.87 \\
Tween 40 & 2.3 \\
Brij 35 & 1.2 \\
Cetostearyl alcohol & \\
Co-surfactant & 8.45 \\
Propylene glycol & 3.8 \\
PEG 400 & 1.79 \\
Ethanol & \\
\hline
\end{tabular}

results are similar to that obtained by Saberi et al. (2013), and this may be due to the higher surfactant mixture concentration result in higher surfactant molecules at the interface and lower interfacial tension producing smaller droplet size. Tween 40 Hydrophilic-Lipophilic Balance (HLB $=15.6$ ) is considered a good choice for preparation of NE since it is usually produced the smallest droplets size (Hasani et al., 2015). Average droplet size distribution of formulas produced NE ranged from 54.82 to $80.57 \mathrm{~nm}$. Also, the results showed that the viscosity of NE increased without change in $\mathrm{pH}$ (5.65) as surfactant mixture concentration increases. Although formula F14 has the smallest droplet size, formula (F10) was chosen as an optimum formula for drug loading because of lowest viscosity and low surfactant mixture percentage gave nanodroplet size NE.

\section{Evaluation of prepared nanoemulsion-enriched hydrogel of EC}

The results in Table 5 of six prepared formulas (G1-G6) of the NE-enriched hydrogel of EC indicate no phase separation, acceptable drug content (99.60-98.70), and acceptable $\mathrm{pH}$ range of 5.82-6.52. The viscosity of product showed an increase as the gelling polymer percent increased. Formulas containing HPMC showed higher viscosities than carbopol containing formulas. The spreadability and extrudability of the product showed a decrease as the gelling polymer increased.

\section{In vitro drug release}

The release of drug from the prepared product (Fig. 4) shows an increase in the drug release retardation as carbopol or HPMC percentage increases although HPMC showed higher retardation effect on drug release than carbopol. The results indicated that formula G6 which contains 3.5\% HPMC has an extended but complete drug release a period of 8 hours and can be considered as the best formula of NE-enriched hydrogel with good physical properties.

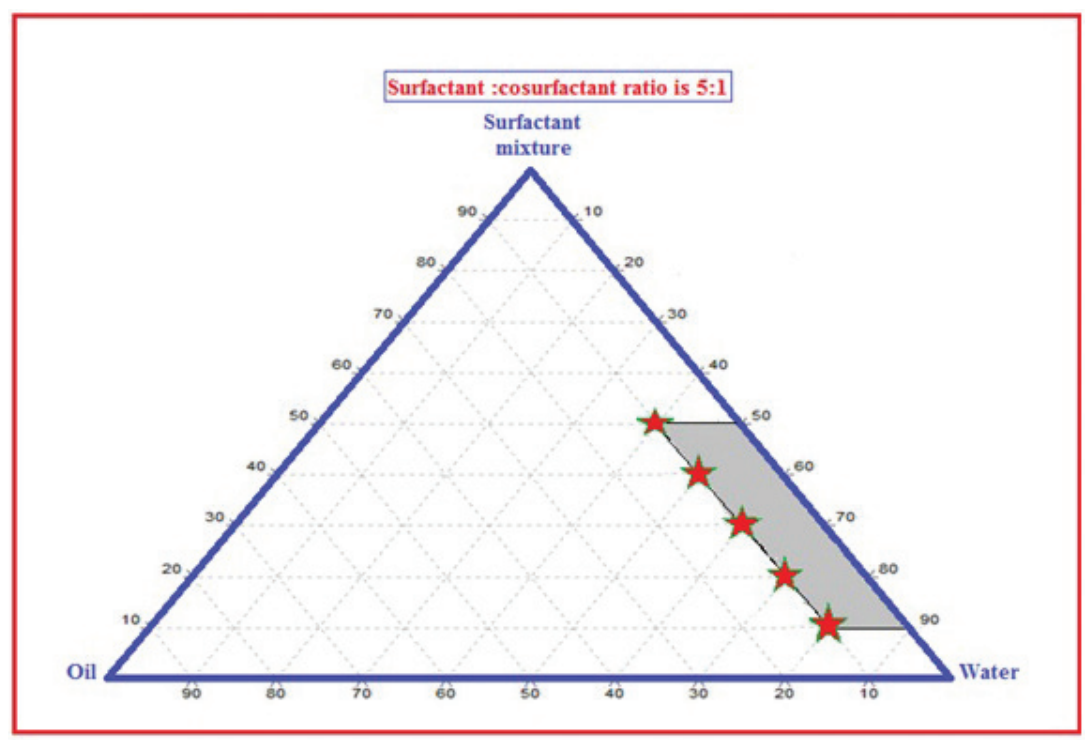

Figure 3. Pseudo-ternary phase diagram of peppermint oil: Tween 40/propylene glycol: water exploring the nanoemulsion region as marked points. 
Table 4. Physical properties of the prepared systems which are composed of peppermint oil: Tween 40/propylene glycol: water.

\begin{tabular}{|c|c|c|c|c|c|}
\hline Formulas code & Appearance & Mean particle size (nm) & Viscosity (CPS) & Polydispersity index (PDI) & $\mathrm{pH}$ \\
\hline $\mathrm{F} 1$ & Turbid & & & & \\
\hline F2 & Turbid & & & & \\
\hline F3 & Turbid & & & & \\
\hline $\mathrm{F} 4$ & Turbid & & & & \\
\hline F5 & Turbid & & & & \\
\hline F6 & Turbid & & & & \\
\hline F7 & Turbid & & & & \\
\hline F8 & Turbid & & & & \\
\hline F9 & Turbid & & & & \\
\hline F10 & Clear & 80.57 & 0.82 & 0.083 & 5.65 \\
\hline F11 & Clear & 75.44 & 0.85 & 0.073 & 5.65 \\
\hline $\mathrm{F} 12$ & Clear & 66.21 & 0.86 & 0.072 & 5.65 \\
\hline F13 & Clear & 62.73 & 0.89 & 0.069 & 5.65 \\
\hline F14 & Clear & 54.82 & 0.99 & 0.041 & 5.65 \\
\hline
\end{tabular}

Table 5. Evaluation parameters of nanoemulsion-enriched hydrogel of EC.

\begin{tabular}{ccccccc}
\hline Formulas code & Phase separation & $\mathbf{p H}$ & Viscosity (CPs) & Spreadability (g.cm/sec) & Extrudability (g/sec) & Drug content (\%) \\
\hline G1 & No & 5.82 & 1250 & 35 & 1.7 & 99.60 \\
G2 & No & 5.85 & 1360 & 33 & 3.5 & 98.40 \\
G3 & No & 5.91 & 1730 & 50 & 1.3 & 98.40 \\
G4 & No & 6.35 & 5260 & 20 & 0.9 & 99.30 \\
G5 & No & 6.47 & 6470 & 19 & 0.8 & 99.73 \\
G6 & No & 6.52 & 7200 & 18 & 0.7 & 98.70 \\
\hline
\end{tabular}

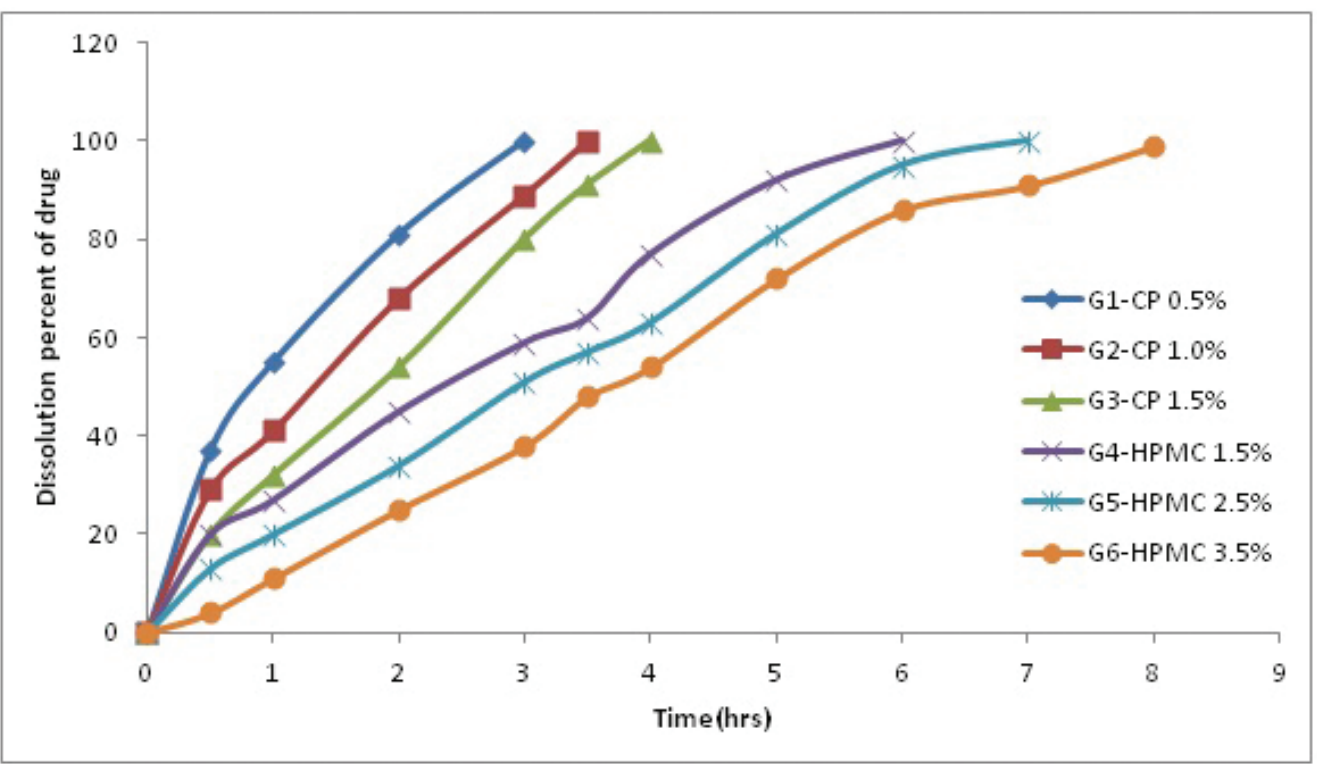

Figure 4. The dissolution profile of EC from NE-enriched with carbopol (G1-G3) or with HPMC (G4-G6). 


\section{CONCLUSION}

Results of this study suggest that formula of nanosized topical product with the complete and extended release of poorly soluble antifungal drug can be prepared with expected higher penetration through the skin due to small particle size.

\section{REFERENCES}

Ahmed K, Li Y, McClements DJ, Xiao H. Nanoemulsion-and emulsion-based delivery systems for curcumin: encapsulation and release properties. Food Chem, 2012; 132(2):799-807.

Amidon GL, Lennernäs H, Shah VP, Crison JR. A theoretical basis for a biopharmaceutic drug classification: the correlation of in vitro drug product dissolution and in vivo bioavailability. Pharm Res, 1995; 12(3):413-20.

Baibhav, J, Gurpreet S, Rana AC, Seema S. Development and characterization of clarithromycin emulgel for topical delivery. Int J Drug Dev Res, 2012; 4(3):310-23.

Cavrini, V, Di Pietra AM, Raggi MA. High-pressure liquid chromatographic (HPLC) analysis of imidazole antifungals in commercial dosage forms. Int J Pharm, 1982; 10(2):119-24.

El AE, Soliman OA, El-Dahan MS, Al-Zuhairy SA. Improvement of the ocular bioavailability of econazole nitrate upon complexation with cyclodextrins. AAPS PharmSciTech, 2017; 18(5):1795-809.

Ge S, Lin Y, Lu H, Li Q, He J, Chen B, Wu C, Xu Y. Percutaneous delivery of econazole using microemulsion as vehicle: formulation, evaluation and vesicle-skin interaction. Int J Pharm, 2014; 465(1-2):120-31.

Gorain B, Choudhury H, Kundu A, Sarkar L, Karmakar S, Jaisankar P, Pal TK. Nanoemulsion strategy for olmesartan medoxomil improves oral absorption and extended antihypertensive activity in hypertensive rats. Colloids Surf B Biointerf, 2014; 115:286-94.

Guttoff M, Saberi AH, McClements DJ. Formation of vitamin D nanoemulsion-based delivery systems by spontaneous emulsification: factors affecting particle size and stability. Food Chem, 2015; 171:117-22.

Hasani, F, Pezeshki A, Hamishehkar H. Effect of surfactant and oil type on size droplets of betacarotene-bearing nanoemulsions. Int J Curr Microbiol App Sci, 2015; 4:146.

Hoare TR, Kohane DS. Hydrogels in drug delivery: progress and challenges. Polymer, 2008; 49(8):1993-2007.

Jadhav, C, Kate V, Payghan SA. Investigation of effect of nonionic surfactant on preparation of griseofulvin non-aqueous nanoemulsion. J Nanostruct Chem, 2015; 5(1):107-13.

Abdul Rasool BK, Gareeb RH, Fahmy SA, Abdul Rasool AA. Meloxicam- $\beta$-cyclodextrin transdermal gel: physicochemical characterization and in vitro dissolution and diffusion studies. Curr Drug Deliv, 2011; 8(4):381-91.
Kumar L, Verma R. In vitro evaluation of topical gel prepared using natural polymer. Int J Drug Deliv, 2010; 2(1):58-63.

Ogata M, Matsumoto H, Hamada Y, Takehara M, Tawara K. 1-[1-[2-[(3-Chlorobenzyl) Oxy] Phenyl] Vinyl]-1H-Imidazole hydrochloride, a new potent antifungal agent. J Med Chem, 1983; 26(5):768-70

Ranga PM, Sellakumar V, Natrajan R, Kumar MK. Formulation and in vitro evaluation of ciprofloxacin loaded topical emulgel. Int J Pharm Chem Sci, 2012; 1:237-42.

Remington JP. 1 Remington: The Science and Practice of Pharmacy. Lippincott Williams \& Wilkins, USA, 2006.

Saberi AH, Fang Y, McClements DJ. Fabrication of vitamin E-enriched nanoemulsions: factors affecting particle size using spontaneous emulsification. J Colloid Interface Sci, 2013; 391:95-102.

Shakeel F, Shafiq S, Haq N, Alanazi FK, Alsarra IA Nanoemulsions as potential vehicles for transdermal and dermal delivery of hydrophobic compounds: an overview. Expert Opin Drug Deliv, 2012; 9(8):953-74.

Sood S, Jain K, Gowthamarajan K. Optimization of curcumin nanoemulsion for intranasal delivery using design of experiment and its toxicity assessment. Colloids Surf B Biointerf, 2014; 113:330-7.

Stahl PH, Wermuth CG. Handbook of pharmaceutical salts: properties, selection and use. Chem Int, 2002; 24:21.

Sugumar S, Clarke SK, Nirmala MJ, Tyagi BK, Mukherjee A, Chandrasekaran N. Nanoemulsion of eucalyptus oil and its larvicidal activity against culex quinquefasciatus. Bull Entomol Res, 2014; 104(3):393-402.

Suñer-Carbó J, Boix-Montañés A, Halbaut-Bellowa L, Velázquez-Carralero $\mathrm{N}$, Zamarbide-Ledesma J, Bozal-de-Febrer $\mathrm{N}$, Calpena-Campmany AC. Skin permeation of econazole nitrate formulated in an enhanced hydrophilic multiple emulsion. Mycoses, 2017; 60(3):166-77.

Vikrant J, Sonali N. Formulation and evaluation of topical flurbiprofen gel using different gelling agents. World J Pharma Sci, 2013; 3(9):654-63.

Zhao L, Wei Y, Huang Y, He B, Zhou Y, Fu J. Nanoemulsion improves the oral bioavailability of baicalin in rats: in vitro and in vivo evaluation. Int J Nanomed, 2013; 8:3769.

How to cite this article:

Ghareeb MM. Design and in vitro characterization of a topical nanoemulsion-enriched hydrogel of econazole nitrate. J Appl Pharm Sci, 2019; 9(01):051-057. 